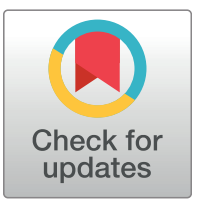

G

Citation: Day F, Karaderi T, Jones MR, Meun C, He C, Drong A, et al. (2019) Correction: Large-scale genome-wide meta-analysis of polycystic ovary syndrome suggests shared genetic architecture for different diagnosis criteria. PLoS Genet 15(12): e1008517. https://doi.org/10.1371/journal. pgen. 1008517

Published: December 5, 2019

Copyright: @ 2019 Day et al. This is an open access article distributed under the terms of the Creative Commons Attribution License, which permits unrestricted use, distribution, and reproduction in any medium, provided the original author and source are credited.
CORRECTION

\section{Correction: Large-scale genome-wide meta- analysis of polycystic ovary syndrome suggests shared genetic architecture for different diagnosis criteria}

Felix Day, Tugce Karaderi, Michelle R. Jones, Cindy Meun, Chunyan He, Alex Drong, Peter Kraft, Nan Lin, Hongyan Huang, Linda Broer, Reedik Magi, Richa Saxena, Triin Laisk, Margrit Urbanek, M. Geoffrey Hayes, Gudmar Thorleifsson, Juan FernandezTajes, Anubha Mahajan, Benjamin H. Mullin, Bronwyn G. A. Stuckey, Timothy D. Spector, Scott G. Wilson, Mark O. Goodarzi, Lea Davis, Barbara Obermayer-Pietsch, André G. Uitterlinden, Verneri Anttila, Benjamin M. Neale, Marjo-Riitta Jarvelin, Bart Fauser, Irina Kowalska, Jenny A. Visser, Marianne Andersen, Ken Ong, Elisabet Stener-Victorin, David Ehrmann, Richard S. Legro, Andres Salumets, Mark I. McCarthy, Laure MorinPapunen, Unnur Thorsteinsdottir, Kari Stefansson, the 23andMe Research Team, Unnur Styrkarsdottir, John R. B. Perry, Andrea Dunaif, Joop Laven, Steve Franks, Cecilia M. Lindgren, Corrine K. Welt

In the Data Availability Statement, the URL does not fully match the data described. The URL should be: https://doi.org/10.17863/CAM.36024. The correct Data Availability Statement is: Summary statistic GWAS meta-analysis results for the combined dataset excluding 23andMe are available at https://doi.org/10.17863/CAM.36024. The most significant 10,000 SNPs for the meta-analysis including 23andMe are available at https://doi.org/10.17863/CAM.36024.

There is an error in Table 2. The square brackets that indicate the effect allele are shown in error. Please see the correct Table 2 here.

S2 Table is a duplicate of S6 Table. Please view the correct S2 Table below. 
Table 2. The 14 genome-wide significant variants associated with PCOS in the meta-analysis.

\begin{tabular}{|c|c|c|c|c|c|c|c|c|c|c|c|c|}
\hline Chr. Position $^{1}$ & rsID & $\mathbf{E A}^{2}$ & $\mathbf{O A}^{3}$ & EAF $^{4}$ & Beta & $\mathrm{OR}^{5}$ & $95 \% \mathrm{CI}^{6}$ & Std. Error & Nearest Gene & P-value & Effective $\mathbf{N}^{7}$ & $\operatorname{Ref}^{8}$ \\
\hline $2: 43561780$ & rs7563201 & A & G & 0.451 & -0.108 & 0.90 & $(0.86 \pm 0.92)$ & 0.0172 & THADA & $3.68 \mathrm{e}-10$ & 17192 & \\
\hline $2: 213391766$ & rs2178575 & A & G & 0.151 & 0.166 & 1.18 & $(1.13 \pm 1.23)$ & 0.0219 & $E R B B 4$ & $3.34 \mathrm{e}-14$ & 17192 & 17 \\
\hline 5:131813204 & rs13164856 & $\mathrm{T}$ & C & 0.729 & 0.124 & 1.13 & $(1.08 \pm 1.17)$ & 0.0193 & IRF1/ RAD50 & $1.45 \mathrm{e}-10$ & 17192 & 17 \\
\hline $8: 11623889$ & rs804279 & A & $\mathrm{T}$ & 0.262 & 0.128 & 1.14 & $(1.09 \pm 1.17)$ & 0.0184 & GATA4/ NEIL2 & $3.76 \mathrm{e}-12$ & 16895 & 16 \\
\hline 9:5440589 & rs10739076 & A & C & 0.308 & 0.110 & 1.12 & $(1.07 \pm 1.15)$ & 0.0197 & PLGRKT & $2.51 \mathrm{e}-08$ & 17192 & \\
\hline 9:97723266 & rs7864171 & A & G & 0.428 & -0.093 & 0.91 & $(0.88 \pm 0.94)$ & 0.0168 & FANCC & $2.95 \mathrm{e}-08$ & 17192 & 16 \\
\hline $9: 126619233$ & rs9696009 & A & G & 0.068 & 0.202 & 1.22 & $(1.15 \pm 1.30)$ & 0.0311 & DENND1A & $7.96 \mathrm{e}-11$ & 17192 & \\
\hline $11: 30226356$ & rs11031005 & $\mathrm{T}$ & C & 0.854 & -0.159 & 0.85 & $(0.81 \pm 0.89)$ & 0.0223 & $A R L 14 E P / F S H B$ & $8.66 \mathrm{e}-13$ & 17192 & 16,17 \\
\hline 11:102043240 & rs11225154 & A & G & 0.094 & 0.179 & 1.20 & $(1.13 \pm 1.26)$ & 0.0272 & $Y A P 1$ & $5.44 \mathrm{e}-11$ & 17192 & 17 \\
\hline 11:113949232 & rs1784692 & $\mathbf{T}$ & C & 0.824 & 0.144 & 1.15 & $(1.10 \pm 1.20)$ & 0.0226 & ZВТВ16 & $1.88 \mathrm{e}-10$ & 17192 & \\
\hline $12: 56477694$ & rs2271194 & A & $\mathrm{T}$ & 0.416 & 0.097 & 1.10 & $(1.06 \pm 1.13)$ & 0.0166 & $E R B B 3 / R A B 5 B$ & $4.57 \mathrm{e}-09$ & 17192 & 17 \\
\hline $12: 75941042$ & rs1795379 & $\mathrm{T}$ & C & 0.240 & -0.117 & 0.89 & $(0.85 \pm 0.92)$ & 0.0195 & $K R R 1$ & $1.81 \mathrm{e}-09$ & 17192 & 17 \\
\hline $16: 52375777$ & rs8043701 & A & $\mathrm{T}$ & 0.815 & -0.127 & 0.88 & $(0.84 \pm 0.91)$ & 0.0208 & TOX3 & $9.61 \mathrm{e}-10$ & 17192 & \\
\hline $20: 31420757$ & rs853854 & A & $\mathbf{T}$ & 0.499 & -0.098 & 0.91 & $(0.87 \pm 0.93)$ & 0.0163 & MAPRE1 & $2.36 \mathrm{E}-09$ & 17192 & \\
\hline
\end{tabular}

1. Chromosome and position in hg19.

2. EA-Effect allele.

3. OA-Other allele.

4. EAF-Effect allele frequency.

5. OR-Odds Ratio.

6. 95\% CI- 95 percent confidence interval.

7. Effective N-effective sample size.

8. Ref.-reference.

\section{Supporting information}

S2 Table. All PCOS meta-analysis, PCOS meta-analysis without self-report, NIH, nonNIH Rotterdam and self-report meta-analysis results.

(XLSX)

\section{Reference}

1. Day F, Karaderi T, Jones MR, Meun C, He C, Drong A, et al. (2018) Large-scale genome-wide metaanalysis of polycystic ovary syndrome suggests shared genetic architecture for different diagnosis criteria. PLoS Genet 14(12): e1007813. https://doi.org/10.1371/journal.pgen.1007813 PMID: 30566500 